\title{
BM] Global Health Epidemiology and outcomes of Clostridium difficile infection among hospitalised patients: results of a multicentre retrospective study in South Africa
}

\author{
Laurel Legenza, ${ }^{1}$ Susanne Barnett, ${ }^{1}$ Warren Rose, ${ }^{1}$ Monica Bianchini, ${ }^{1}$ \\ Nasia Safdar, ${ }^{2}$ Renier Coetzee ${ }^{3}$
}

To cite: Legenza L, Barnett S, Rose W, et al. Epidemiology and outcomes of Clostridium difficile infection among hospitalised patients: results of a multicentre retrospective study in South Africa. BMJ Glob Health 2018;3:e000889. doi:10.1136/ bmjgh-2018-000889

\section{Handling editor Sanni Yaya}

Received 10 April 2018 Revised 31 May 2018 Accepted 16 June 2018
Check for updates

(c) Author(s) (or their employer(s)) 2018. Re-use permitted under CC BY-NC. No commercial re-use. See rights and permissions. Published by BMJ.

${ }^{1}$ School of Pharmacy, University of Wisconsin-Madison, Madison, Wisconsin, USA

${ }^{2}$ School of Medicine and Public Health, University of Wisconsin, Madison, Wisconsin, USA

${ }^{3}$ School of Pharmacy, University of the Western Cape, Cape

Town, South Africa

Correspondence to

Dr Renier Coetzee;

recoetzee@uwc.ac.za

\section{ABSTRACT}

Introduction Limited data exist on Clostridium difficile infection (CDI) in low-resource settings and settings with high prevalence of HIV. We aimed to determine baseline CDI patient characteristics and management and their contribution to mortality.

Methods We reviewed adult patients hospitalised with diarrhoea and a $C$. difficile test result in 2015 from four public district hospitals in the Western Cape, South Africa. The primary outcome measures were risk factors for mortality. Secondary outcomes were $C$. difficile risk factors (positive vs negative) and CDI treatment.

Results Charts of patients with diarrhoea tested for $C$. difficile $(\mathrm{n}=250 ; 112$ C. difficile positive, 138 C. difficile negative) were reviewed. The study population included more women $(65 \%)$. C. difficile-positive patients were older ( 46.5 vs 40.7 years, $p<0.01$ ). All-cause mortality was more common in the $C$. difficile-positive group (29\% vs $8 \%, p<0.0001 ; \mathrm{HR} 2.0,95 \% \mathrm{Cl} 1.1$ to 3.6). Tuberculosis (C. difficile positive $54 \%$ vs $C$. difficile negative $32 \%$, $\mathrm{p}<0.001)$, 30-day prior antibiotic exposure (C. difficile positive $83 \%$ vs $C$. difficile negative $46 \%, p<0.001$ ) and prior hospitalisation (C. difficile positive $55 \%$ vs $C$. difficile negative $22 \%, p<0.001$ ) were also more common in the C. difficile-positive group. C. difficile positive test result (OR 4.7, $95 \% \mathrm{Cl} 2.0$ to $11.2 ; \mathrm{p}<0.001$ ), male gender ( $\mathrm{OR}$ $2.8,95 \% \mathrm{Cl} 1.1$ to $7.2 ; \mathrm{p}=0.031)$ and tuberculosis ( $\mathrm{OR}$ $2.3,95 \% \mathrm{Cl} 1.0$ to $5.0 ; \mathrm{p}=0.038$ ) were independently associated with mortality. Of patients starting treatment, metronidazole was the most common antimicrobial therapy initiated $(70 \%, n=78) ; 32$ C. difficile-positive $(29 \%)$ patients were not treated.

Conclusion Patients testing positive for $C$. difficile are at high risk of mortality at public district hospitals in South Africa. Tuberculosis should be considered an additional risk factor for $\mathrm{CDI}$ in populations with high tuberculosis and HIV comorbidity. Interventions for CDI prevention and management are urgently needed.

\section{INTRODUCTION}

Clostridium difficile infection (CDI) is an increasing global health concern resulting in

\section{Key questions}

What is already known?

- Patients in South Africa have significant comorbidities distinct from high-resource countries, including a higher incidence of HIV and tuberculosis, which may uniquely increase patients' risk for Clostridium difficile infection (CDI).

What are the new findings?

- This study is the first examining risk factors, management, infection control and mortality among hospitalised patients in public hospitals in sub-Saharan Africa to our knowledge.

- The majority of patients treated for CDI received metronidazole, while the mortality of patients with a $C$. difficile positive result was significantly higher than similar patients testing negative with diarrhoea.

- In populations with high tuberculosis and HIV comorbidity, tuberculosis is an additional risk factor for CDI.

What do the new findings imply?

- Vancomycin should be considered as an alternative to metronidazole in populations with high prevalence of tuberculosis and immunocompromising conditions as a high mortality rate was observed in this study.

severe diarrhoea, excessive healthcare costs, readmissions and mortality. Life-threatening complications resulting from CDI include sepsis, pseudomembranous colitis and toxic megacolon. The majority of CDI studies have been conducted in high-resource countries. ${ }^{1} \mathrm{CDI}$ incidence increased yearly in these settings after 2000 until recently; a decline in CDI incidence from 2011 to 2015 in longterm care settings was reported in association with decreased hospital fluoroquinolone use and detection of the NAP1/027 strain. ${ }^{12} C$. difficile remains the most common pathogen 
implicated in hospital-acquired infections in the USA. ${ }^{34}$ CDI in patients is associated with antibiotic use, which leads to disruption of normal flora and uninhibited growth of toxigenic C. difficile. ${ }^{5}$ Antibiotics commonly associated with CDI include fluoroquinolones, third-generation cephalosporins, clindamycin and penicillins. ${ }^{6}$ Advanced age is a noted risk factor for CDI, and commonly associated with CDI mortality in high-resource countries. ${ }^{7}$ Additional risk factors for CDI include hospitalisation, inflammatory bowel disease, immunodeficiency, organ transplantation, chemotherapy, gastric acid suppression, chronic kidney disease and exposure to individuals with C. difficile. ${ }^{6}$ The healthcare environment and patients in low-resource settings are distinct from high-resource settings. For example, South Africa has the lowest life expectancy in the world, 49.7 years, reducing the likelihood for elderly age to be a CDI risk factor. ${ }^{8}$ Meanwhile, the relationship of CDI and the infectious diseases associated with mortality in this population is understudied. Thus, further investigation of CDI risk factors in these countries is urgently needed.

In South Africa, the leading causes of death are infectious diseases including tuberculosis, influenza/pneumonia and HIV, which may uniquely increase patients' risk for CDI. ${ }^{9}{ }^{10}$ South Africa has the largest known HIV epidemic in the world. Adult prevalence is estimated to be $18.9 \%$, and $19 \%$ of people living with HIV globally reside in South Africa. ${ }^{11}$ Tuberculosis incidence in South Africa is the sixth highest globally. Coinfection of tuberculosis in patients with HIV is a synergistic epidemic, including a disproportionate rate of HIV-associated tuberculosis deaths as $63 \%$ of tuberculosis cases are in patients with HIV. ${ }^{12}{ }^{13}$ Over the past decade, tuberculosis has surpassed HIV and cardiovascular disease as the leading cause of death. ${ }^{14}$ While CDI studies in Africa are limited, two previous studies at a tertiary hospital in Cape Town, South Africa, documented 9\%-22\% of patients with diarrhoea tested $C$. difficile positive using different methods. ${ }^{1516}$ In one of these studies, patients with $C$. difficile positive results were associated with antibiotic use in the previous 28 days and hospitalisation within the previous 90 days compared with patients with negative results. ${ }^{15}$ The prevalence of the NAP1/027 strain was $3.4 \%$, which is substantially lower than in high-resource settings such as the USA, which has ranged from a $16.9 \%$ to $26.2 \%$ prevalence in recent studies. ${ }^{15} 17$

Understanding the epidemiology of CDI in low-resource settings is essential to improve identification, prevention and treatment measures. In the present study, we identify patient CDI characteristics and management in resource-limited public district level hospitals and their contribution to mortality.

\section{METHODS}

Local infectious disease leaders were consulted early in study design, starting in August 2015. These leaders identified CDI as a critical public health challenge in
South Africa because of its increasing incidence and high morbidity and mortality globally, lack of local studies and vulnerable populations with HIV and tuberculosis locally. In addition to the lack of CDI studies performed in these hospitals and this patient population, focus on district hospitals was also recommended due to the scarcity of CDI data at this level. Subsequently the University of the Western Cape Research Ethics Committee, National Health Laboratory Service (NHLS) and Western Cape Department of Health granted approval for the first CDI epidemiological study in South African district level hospitals.

On approval, a multicentre, retrospective chart review was conducted at four district-level hospitals, averaging 265 inpatient beds, in Cape Town, South Africa. The study included hospitalised adult patients $(>18$ years of age) with diarrhoea and either a positive or negative $C$. difficile PCR test result from one or more stool samples during the year 2015. These patients were identified from a list of $C$. difficile test results provided by NHLS, which is a national network of diagnostic laboratories that serve $80 \%$ of the South African population, including the Western Cape Department of Health hospitals. All stool samples from the district-level hospitals included in this study were sent to the NHLS laboratory at the nearest tertiary-level hospital. Standardised NHLS protocols indicated PCR testing for all eligible samples in 2015 (NHLS does not perform $C$. difficile tests on solid stool samples or patients with a recent $C$. difficile positive result). All patients included in the study had diarrhoea that took the shape of the container and clinical suspicion for CDI. A minimum number of stools within 24 hours was not required for study inclusion as frequency was inconsistently documented. Any additional aetiologies tested were not included in the laboratory report. Test results originating from paediatric patients, outpatient clinics or day surgery patients were excluded. Patients with tests ordered in the emergency department were included if the patient was subsequently admitted to the hospital.

The primary outcome of this study was the identification of risk factors for mortality in South African patients in the Western Cape with diarrhoea. Secondary outcomes were risk factors for a $C$. difficile positive result compared with a $C$. difficile negative result, and within this group, risk factors for mortality and management of CDI.

The 2015 medical records for any patient with a C. difficile test result were reviewed from August 2016 to April 2017. Data collection was subject to the available processes for medical record review and availability of files at each individual hospital. Paper folders were requested from the medical records department at each hospital and reviewed by study personnel onsite. At one hospital, medical records were accessible electronically by access granted to view scanned files of the patient folders remotely. The review included all available patient records with a positive $C$. difficile test result. At each hospital, an equal number of $C$. difficile-positive and negative patient charts were requested and reviewed 
if available. At most hospitals, the number of negative test results was much larger than positive results. Therefore, all identified patients with a positive $C$. difficile test result were requested, while patients with a negative test result were randomly selected following an autogenerated random number process. The randomisation of the negative chart numbers was performed to address selection bias. If the total number of patients tested at each hospital was less than 25, all available charts were reviewed. As this is the first epidemiologic study in district-level hospitals, the magnitude of tests was difficult to predict. In this study design, the number of positive results limited sample size during the year evaluated and an a priori sample size calculation was not performed.

Use of a structured data collection tool allowed for review of all clinical and laboratory notes available in the medical record from the hospital admission including the $C$. difficile test. Pertinent records prior to the admission were also reviewed using the same tool to determine medical history and previous antibiotic exposure, and postadmission records were reviewed to determine patient outcomes, recurrence and mortality. Data collected included demographics (gender, age, allergies), comorbid conditions (HIV, tuberculosis, multidrug-resistant tuberculosis, diabetes, cardiovascular conditions: heart failure, hypertension, hyperlipidaemia, other cardiovascular conditions, malignancy, inflammatory bowel diseases: ulcerative colitis, Crohn's, other immunocompromising condition), hospitalisations prior to the current admission (0-30 and 31-90 days prior to test order) and previous antibiotic exposure, including a single dose (penicillins, quinolones, carbapenems, cephalosporin, clindamycin or other), and indication in prior 30 and 90 days from date of written CDI order, CDI history (current episode is documented as first CDI episode, recurrence, unknown if first episode or recurrence), clinical presentation (diarrhoea, temperature $\left(>38^{\circ} \mathrm{C}\right)$, haematochezia, pseudomembranous colitis), dates of admission, rehydration, loperamide use, CDI antibiotic treatment, CDI-related infection control (isolation and contact precautions) and reason for hospitalisation.

Data were analysed using Stata SE statistical software (V.15.0, StataCorp, College Station, Texas, USA). Summary statistics for infection management were determined, including antibiotic treatment and infection prevention and control components. Length of stay (LOS) was summarised and compared by t-tests both by C. difficile test result and hospital mortality to express mean, median and statistically significant differences. Univariate summary statistics were calculated for age and gender of individual patients. $\mathrm{X}^{2}$ tests were conducted by $C$. difficile test result and mortality for patient characteristics and CDI risk factors. A survival analysis with the Gehan-Breslow-Wilcoxon test was determined for patients with $C$. difficile positive PCR versus $C$. difficile negative PCR in GraphPad Prism (V.6, GraphPad Software, La Jolla, California, USA), with a start date equal to when an order for $C$. difficile test was written and end date the day of mortality or discharge. Censoring occurred for patients discharged before 30 days to account for uncertainty of survival and readmission after discharge. Patients with a clinic visit or hospital admission occurring greater than 30 days after the $C$. difficile test were categorised as survivors. $\mathrm{P}$ values $\leq 0.05$ were considered significant. All variables identified as at least marginally significant $(\mathrm{p}<0.10)$ predictors in the univariate mortality analysis were included in the model. Independent predictors of all-cause mortality were determined via a separate multivariable logistic regression.

\section{RESULTS}

Overall, 652 C. difficile PCR tests were conducted in 2015 from the four hospitals included; 19 of these had an error result and were excluded. Forty-one of the 291 patient charts requested were excluded because either the patient chart was unavailable or missing, the chart lacked adequate documentation to review related to the test date, or the test met exclusion criteria for not occurring during a hospitalisation (eg, outpatient clinic, day surgery). Of 139 positive results, 112 results and corresponding charts were reviewed and of 494 negative results, 138 negative results were reviewed (total $n=250$; figure 1). Two tests were reviewed for one patient during a 76-day LOS. All other test results reviewed were from unique hospitalisations. The 250 test results reviewed represent 225 individual patients.

Patient characteristics analysed by test result in the univariate analyses are presented in table 1 . Significant differences were found in the patient demographics in patients testing positive versus negative for $C$. difficile including age, tuberculosis, prior hospitalisation and specific antibiotic use. Mean age and gender distribution was calculated for 225 individual patients (102 C. difficile+; 123 C. difficile-), excluding subsequent test results from patients with more than one test in the study period. The mean age for the $C$. difficile-positive patients was 46.5 years compared with 40.7 years for $C$. difficile-negative patients $(p<0.01)$. There were more women in the study population $(65 \%)$. However, mean age was similar between men and women in this study overall (43.4 vs 43.3 years, respectively).

Presence of comorbid infectious diseases also proved to be a significant variable, particularly with regard to tuberculosis. More patients testing positive for $C$. difficile also had tuberculosis (C. difficile positive $54 \%$ vs $C$. difficile negative $32 \%, \mathrm{p}<0.001)$. HIV rates were high in both C. difficile-positive and C. difficile-negative patients $(71 \%$ vs $80 \%$, respectively; $\mathrm{p}=0.07$ ). Of HIV-positive patients with a $C$. difficile test result, the majority had CD4 counts consistent with AIDS $\left(\mathrm{CD} 4<0.2 \times 10^{\wedge} 9\right.$ cells $/ \mathrm{L} ; C$. difficile positive $81 \%$; C. difficile negative $81 \%$; $\mathrm{n}=178$ ).

Analysis of $C$. difficile test results confirmed prior hospitalisation and antibiotic exposure are important CDI risk factors among patients included in the study. Recent prior hospitalisation was more common in C. difficile-positive 


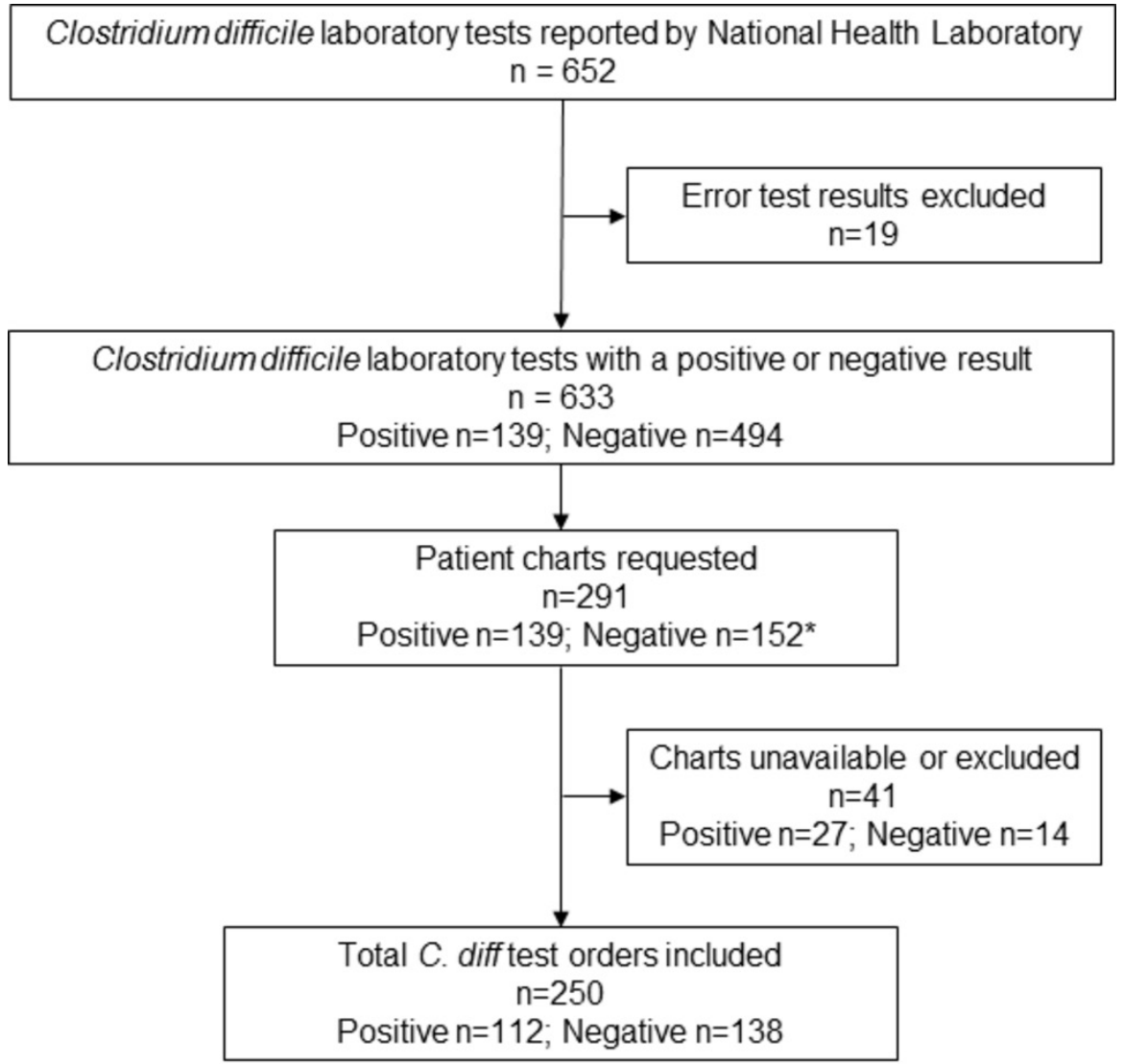

*Number of negatives requested to match number of positive results, plus additional negative results at hospital meeting criteria of $<25$ total records $(152=139+13)$.

Figure 1 Patient Clostridium difficile test result inclusion.

patients for both hospitalisation 30 and 90 days prior to the admission $(\mathrm{p}<0.001)$. Thirty-day prior antibiotic exposure to all antibiotic classes reviewed was significantly higher in the C. difficile-positive group (table 1 ). The most common antibiotic class with recent prior exposure to patients tested for $C$. difficile was cephalosporins, with half of $C$. difficile-positive patients receiving a cephalosporin in the 30 days prior to the $C$. difficile test order (vs $34 \%$ in C. difficile negative, $\mathrm{p}<0.02$ ). Documentation of tuberculosis antibiotic treatment prior to admission was insufficient to report as prior tuberculosis treatment was not consistently detailed for patients with treatment ordered in hospital. Prescriptions outside the hospital and onsite clinic were not captured if not noted in the admission clinical notes.

Mortality in patients with diarrhoea was more common in the C. difficile-positive group (29\% vs $8 \%, \mathrm{p}<0.0001)$. A Kaplan-Meier survival analysis $(p=0.0087)$ for patients evaluated following a $C$. difficile test order (figure 2) found an all-cause mortality HR of 2.0 (95\% CI 1.1 to 3.6) in patients with a $C$. difficile positive test. All mortality identified occurred in-hospital.

Variables with marginal associations $(p<0.01)$ with mortality are presented in table 2 . A logistic regression including these variables, 30-day mortality, $C$. difficile test result, prior hospitalisation ( 30 and 90 days), critical care admission, tuberculosis, sex, multidrug-resistant tuberculosis and haematochezia was performed (table 3) for mortality as a dependent variable. HIV, immunosuppression and malignancy did not meet criteria for inclusion in the model. Multidrug-resistant tuberculosis perfectly predicted mortality, so the variable was dropped from the model $(n=12)$. An independent risk of mortality in patients with diarrhoea with a $C$. difficile positive test result versus $C$. difficile negative test (OR 4.7, 95\% CI 2.0 to $11.2 ; \mathrm{p}<0.001$ ) was found. Clinically meaningful independent variables associated with mortality also included comorbid tuberculosis (OR 2.3, 95\% CI 1.0 to $5.0 ; \mathrm{p}=0.038$ ) and male sex (OR 2.8, 95\% CI 1.1 to 7.2; $\mathrm{p}=0.031$ ). Prior antibiotic exposure overall or with any specific antibiotic class and hospitalisation were not independently associated with mortality.

Components of C. difficile management for the C. difficile-positive patients were assessed $(\mathrm{n}=112)$. Intravenous rehydration was widely provided $(95 \%)$ but oral rehydration was rarely documented $(12 \%)$. Contact precautions were documented for $36 \%$ of patients. Of the $21 \%$ of patients with a $C$. difficile positive result who were allocated to an isolation room, 16 of these 24 patients $(67 \%)$ were also diagnosed with comorbid tuberculosis. 
Table 1 Patient characteristics

\begin{tabular}{|c|c|c|c|}
\hline & Clostridium diffic & poratory test result & \\
\hline & Positive $(n=112)$ & Negative $(n=138)$ & $P$ values \\
\hline Patient characteristic & & & \\
\hline Age average (years) ${ }^{\star}$ & 47 & 40 & $<0.01$ \\
\hline Sex (female) $)^{\star}$ & $68 \%$ & $63 \%$ & 0.43 \\
\hline Documented HIV & $71 \%$ & $80 \%$ & 0.07 \\
\hline CD4 $0.2 \times 10^{\wedge} 9$ cells/L † & $81 \%$ & $81 \%$ & 0.96 \\
\hline Documented tuberculosis & $54 \%$ & $32 \%$ & $<0.001$ \\
\hline Multidrug-resistant tuberculosis & $9 \%$ & $3 \%$ & 0.04 \\
\hline Prior exposure to each $C$. difficile test & & & \\
\hline Hospitalised 30 days prior to admission & $52 \%$ & $22 \%$ & $<0.001$ \\
\hline Hospitalised 31-90 days prior to admission & $44 \%$ & $23 \%$ & 0.001 \\
\hline 30-day antibiotic exposure & $83 \%$ & $46 \%$ & $<0.001$ \\
\hline Penicillin & $21 \%$ & $11 \%$ & 0.02 \\
\hline Quinolone & $25 \%$ & $10 \%$ & $<0.01$ \\
\hline Carbapenem & $22 \%$ & $4 \%$ & $<0.001$ \\
\hline Cephalosporin & $50 \%$ & $34 \%$ & $<0.01$ \\
\hline Clindamycin & $4 \%$ & $0 \%$ & 0.03 \\
\hline 31-90 days of antibiotic exposure & $29 \%$ & $5 \%$ & $<0.001$ \\
\hline Penicillin & $12 \%$ & $1 \%$ & 0.001 \\
\hline Quinolone & $8 \%$ & $1 \%$ & 0.01 \\
\hline Carbapenem & $2 \%$ & $0 \%$ & 0.12 \\
\hline Cephalosporin & $13 \%$ & $3 \%$ & $<0.01$ \\
\hline Clindamycin & $0 \%$ & $0 \%$ & \\
\hline
\end{tabular}

*Mean age and gender distribution calculated for 225 individual patients, excluding patients with more than one test (102 C. difficile+; 123 C. difficile-).

†Of HIV+ patients, patient CD4 counts were available for 178 C. difficile test results (74 C. difficile+; 104 C. difficile-).

Loperamide, contraindicated in CDI, was administered to $44 \%$ of all patients reviewed, which includes $41 \%$ of $C$. difficile-positive and $46 \%$ of $C$. difficile-negative patients. Loperamide was discontinued after documentation of a C. difficile positive result in $22 \%$ of these patients.

Twenty-nine percent of $C$. difficile-positive patients did not have documented treatment. Explanations observed for lack of treatment included patient improvement $(n=2$, $15 \%$ ), no follow-up or documentation of $C$. difficile test result during admission while the result was finalised on a date the patient was still hospitalised $(n=8,25 \%)$, patient discharge or transfer before test result finalised $(n=13$, $41 \%)$ and mortality before result finalised $(n=6,19 \%)$. Metronidazole was the most common antimicrobial

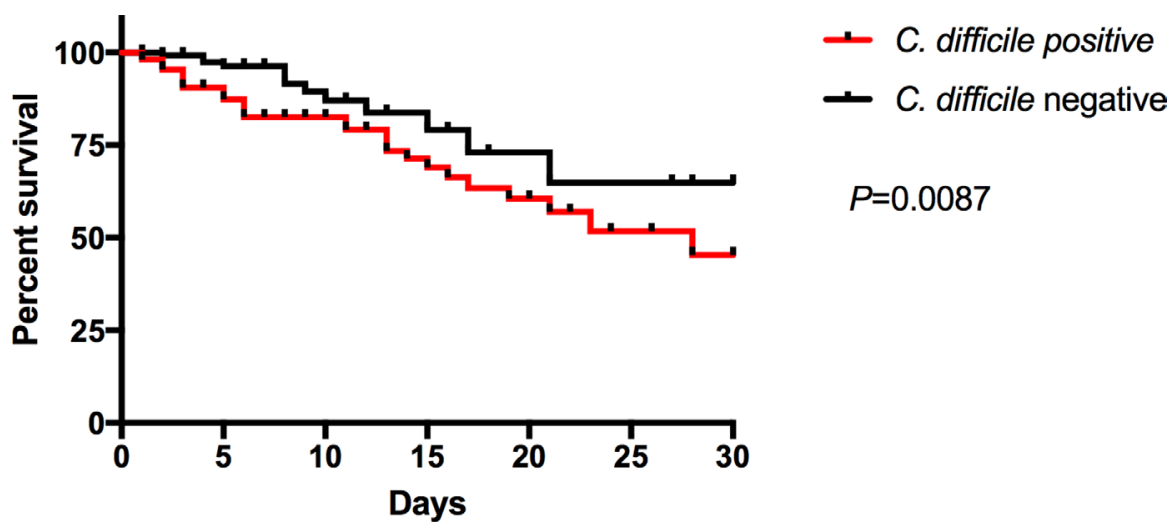

Figure 2 Survival curve for hospitalised patients with diarrhoea following a Clostridium difficile test. HR 2.0 (95\% Cl 1.1 to 3.6). 
Table 2 Univariate analysis of risk factors for mortality found to be marginally significant $(p<0.1)$

\begin{tabular}{ll}
\hline Variables & P values \\
\hline Clostridium difficile test result & 0.000 \\
Hospitalised 30 days prior to admission & 0.004 \\
Critical care admission & 0.014 \\
Tuberculosis & 0.046 \\
Gender & 0.051 \\
Multidrug-resistant tuberculosis & 0.085 \\
Hospitalised 90 days prior to admission & 0.094 \\
Haematochezia & 0.097 \\
\hline
\end{tabular}

therapy initiated $(70 \%, \mathrm{n}=78)$. Metronidazole strength was most often $400 \mathrm{mg}(95 \%)$ for initial treatment and usually ordered every 8 hours $(97 \%)$, consistent with the South African Standard Treatment Guidelines. ${ }^{18}$ Treatment durations, however, varied from the 10-day guideline recommendation. Duration of initial metronidazole therapy ordered ranged from 5 to 14 days, with 10 days being the most commonly prescribed $(45 \%)$. Metronidazole orders less than or equal to 7 days were written for $30 \%$ of patients. C. difficile antibiotic treatment prescribing rates were not significantly different across the four hospitals.

Two patients were treated with oral vancomycin monotherapy for initial treatment. Vancomycin was added to or replaced metronidazole treatment in $14.3 \%$ of patients $(n=16)$. When oral vancomycin was added, the frequency of administration was consistent with every 6 hours as per South African Standard Treatment Guidelines for CDI in $47 \%$ of orders. ${ }^{18}$ The most common duration of vancomycin was 10 days $(44 \%)$ and ranged from 5 to 15 days $(31 \%<7$ days). All initial CDI treatment was ordered for oral administration. Intravenous vancomycin was added to metronidazole in two patients (dose 600 and $1000 \mathrm{mg}$ once daily), but we were unable to document whether this vancomycin administration might have been for CDI management or another infection. One patient was changed from oral to intravenous metronidazole for three doses, then changed back to oral administration.
Overall, mean LOS for all hospitalisations reviewed was $10.2 \pm 11.0$ days (median 7 days, range $0-76$ days). Mean LOS for $C$. difficile-positive patients discharged from the hospital was significantly longer $(11.3 \pm 10.5$ days, median 9 days) compared with $C$. difficile-negative patients $(8.2 \pm 8.5$ days, median $=6.5$ days, $\mathrm{p}=0.02$ ). Recurrence could not be accurately assessed on all patients due to inconsistent records before and after the admission evaluated.

\section{DISCUSSION}

Although there is a wealth of data on CDI epidemiology and outcomes from high-resource countries, research from low-resource countries is sparse. This analysis and these data are the first examining CDI, risk factors, management and mortality among hospitalised patients in South Africa at district-level hospitals to our knowledge. Understanding how CDI is currently being treated and which patients are at greatest risk in South Africa is the first step to designing and implementing quality improvement interventions. Prevalence of tuberculosis appears to be strongly associated with CDI incidence and to interact with demographic and other risk factors influencing positive $C$. difficile results and mortality. Significantly more patients in our study who tested positive for C. difficile had tuberculosis $(\mathrm{p}<0.001)$. C. difficile positive test result, tuberculosis and male sex were found to be independent risk factors for 30-day mortality in this study. Consistent with known CDI risk factors in high-resource settings, prior hospitalisation and antibiotic exposure were strongly associated with a positive $C$. difficile test result. Tuberculosis should be considered a risk factor for CDI in this population, as associations and mortality outcomes in this study are revelatory. Tuberculosis is a less critical risk factor in high-resource settings where prevalence is low. Targeted CDI interventions may improve the high mortality identified and apply to similar low-resource settings in the future.

A post hoc power analysis of $C$. difficile result and mortality for our sample size, 112 C. difficile positive results and 138 C. difficile negative results was performed. The calculated effect size of 0.27 indicated we had $99 \%$ power to detect this difference. However, it may be

Table 3 Logistic regression analysis of 30-day mortality

\begin{tabular}{|c|c|c|c|c|}
\hline Variable & OR & SE & $P$ values & $95 \% \mathrm{Cl}$ \\
\hline Clostridium difficile test result & 4.7 & 2.1 & 0.000 & 2.0 to 11.2 \\
\hline Hospitalised 30 days prior to admission & 0.97 & 0.42 & 0.952 & 0.42 to 2.3 \\
\hline Hospitalised 90 days prior to admission & 1.2 & 0.51 & 0.676 & 0.51 to 2.7 \\
\hline Critical care admission & 13.8 & 17.9 & 0.044 & 1.0 to 176 \\
\hline Tuberculosis & 2.3 & 0.91 & 0.038 & 1.0 to 5.0 \\
\hline Gender & 2.8 & 1.3 & 0.031 & 1.1 to 7.2 \\
\hline Multidrug-resistant tuberculosis & 1 & - & - & - \\
\hline Haematochezia & 0.14 & 0.15 & 0.069 & 0.02 to 1.2 \\
\hline
\end{tabular}


difficult to extrapolate these findings to patients treated in the private sector in South Africa, where HIV and tuberculosis prevalence is significantly lower. A weakness of this study includes the limitations of the retrospective design and data. The chart review included primarily handwritten clinical notes that occasionally required interpretation; assistance from local collaborators and study team members was essential in the data collection phase. The data collection was also limited to only information included in the patient charts. For example, prior antibiotics and hospitalisations at institutions other than a patient's local hospital would be missing, as would any paper records not properly combined or available during data collection. Furthermore, information bias from any missing data regarding severe infections or severe comorbidities such as cancer could affect the results. The study data are insufficient to delineate if the association of $\mathrm{CDI}$ and tuberculosis is due to disease pathogenesis or antibiotics administered for tuberculosis. Patients were unable to be evaluated for severe disease as defined by CDI consensus guidelines as laboratory data were often limited in this retrospective review. It is possible, however, that many patients in this study may have had severe CDI as evidenced by the high mortality rate of $C$. difficile-positive patients, independent of other variables. Despite the limitations of this study, we are reporting novel, needed and independently associated factors of significance.

The results of this study indicate key differences relating to CDI risk factors and mortality between high and low-resource countries, specifically regarding associations of CDI to tuberculosis, sex, age and antibiotic exposure. First, tuberculosis is not commonly included in a list of risk factors for CDI, as this infection is relatively infrequent in high-resource countries where CDI has been most studied. The associations of tuberculosis and CDI could be related to prior healthcare exposure and the use of second-line tuberculosis antibiotics, including fluoroquinolones. A study conducted at tertiary hospitals in South Korea found an increased risk of mortality in patients with concomitant CDI and tuberculosis, compared with CDI alone. ${ }^{19}$ This suggests there may be a pathophysiologic or antibiotic-induced relationship between CDI and mortality in patients with tuberculosis. Second, data on sex/gender differences in CDI-associated mortality are limited. Two previous studies implicate male sex with CDI and complications. The first is a study limited to a single centre in France identifying male sex as a predictor of severe CDI. Another is a study that reported male patients with proton pump inhibitor use have a higher risk of CDI mortality. ${ }^{20}{ }^{21}$ Tuberculosis incidence is higher in men in South Africa (male 226000 vs female 154000 ) and a tendency for men to present for healthcare later in their disease course has been reported in South Africa and globally. ${ }^{22} 23$ Despite the propensity for CDI-related mortality in men, previous studies in high-resource settings report women are more likely to have $\mathrm{CDI}^{7}{ }^{24}$ Therefore, distinct CDI-associated risks may exist between men and women. Third, tuberculosis infection may also be a factor in the age of patients hospitalised with CDI in low-resource settings. The relatively young average age of patients included in this study likely reflects the population burden of high tuberculosis incidence in South Africa in the age range of 25-44 years..$^{22}$ Finally, the antibiotic exposure observed in this study resembles patterns observed in England prior to implementing prescribing patterns to control CDI, an element of antimicrobial stewardship. ${ }^{25}$ In addition to continued strengthening of antimicrobial stewardship efforts in South Africa, significant differences discussed in this manuscript highlight the need for population-tailored CDI guidelines, including identification of population-specific CDI risk factors and interventions.

Using the results of this study, clinicians and policymakers in areas with a high prevalence of tuberculosis and HIV should carefully evaluate which patients are at highest risk for a poor outcome from CDI and ensure appropriate initial treatment. The high mortality associated with CDI in this study also highlights the need for prompt identification, appropriate treatment, and infection prevention and control measures in populations with high HIV and tuberculosis prevalence. The 2010 CDI Infectious Diseases Society of America guidelines recommended first-line therapy with oral metronidazole for mild to moderate disease. Since publication of these guidelines, further studies have supported the use of vancomycin as a first-line therapy. Subsequently, the 2017 guidelines now restrict metronidazole to initial non-severe CDI, when other therapies are contraindicated or unavailable. ${ }^{26}$ The majority of patients treated for CDI in this study received metronidazole, and were switched to vancomycin only if initial therapy with metronidazole was proven ineffective. The high mortality rate of patients with CDI in our study suggests that South African patients may benefit from first-line vancomycin therapy as this medication has been shown to have higher clinical cure rates and significantly lower risk of mortality in severe CDI. ${ }^{27}{ }^{28}$ If oral vancomycin therapy is not adopted as first-line, CDI antibiotic treatment decisions should include markers beyond white cell count, especially for immunocompromised patients and in settings where laboratory results are limited. Risk classifications for severe CDI per the European Society of Clinical Microbiology and Infectious Diseases include older age $(>65)$, serious comorbidity, immunodeficiency and intensive care unit admission. ${ }^{29}$

\section{CONCLUSIONS}

This study provides valuable information to healthcare providers, hospital administrators and policymakers regarding the demographics of hospitalised patients with CDI and CDI-associated patient mortality. Tuberculosis comorbidity should be considered a risk factor for CDI in addition to antibiotic use and prior healthcare exposure in populations with high tuberculosis and HIV comorbidity. Patients testing positive for $C$. difficile have 
a significantly higher and independent risk of mortality compared with patients with diarrhoea testing negative at public district hospitals in South Africa. These results can be used to identify patients at risk of developing CDI and to improve the quality of care provided to patients with CDI in similar settings. Our results indicate improved CDI prevention, assessment and management is urgently needed in the Western Cape province.

Acknowledgements We thank the South African Department of Health for their collaboration and the National Health Laboratory Service for providing laboratory data. We also thank the Western Cape hospitals participating in this study. We appreciate the ongoing collaboration between the University of the Western Cape and the University of Wisconsin-Madison

Contributors LL designed the study, designed data collection, monitored data collection for the whole study, collected data, wrote the statistical analysis plan, cleaned and analysed the data, and drafted and revised the paper. She is the guarantor of the study. SB, WR and NS provided guidance on the study and revised the paper. MB designed data collection tools, collected data and revised the paper. $\mathrm{RC}$ facilitated the collaborative project between the University of the Western Cape and the University of Wisconsin, provided guidance on the study and revised the paper.

Funding There was no funding for this study. LL is supported by an internal endowment at the University of Wisconsin-Madison.

Disclaimer The lead author affirms that the manuscript is an honest, accurate and transparent account of the study being reported; that no important aspects of the study have been omitted; and that any discrepancies from the study as planned (and, if relevant, registered) have been explained.

Competing interests None declared.

Patient consent The Ethics Committee did not require consent for this retrospective study and quality improvement project.

Ethics approval The study was approved by the University of the Western Cape Department of Research Development, Ethics Reference No HS/16/1/24.

Provenance and peer review Not commissioned; externally peer reviewed.

Data sharing statement № additional data available. Statistical code available upon request.

Open access This is an open access article distributed in accordance with the Creative Commons Attribution Non Commercial (CC BY-NC 4.0) license, which permits others to distribute, remix, adapt, build upon this work non-commercially, and license their derivative works on different terms, provided the original work is properly cited, appropriate credit is given, any changes made indicated, and the use is non-commercial. See:@http://creativecommons.org/licenses/by-nc/4.0/.

\section{REFERENCES}

1. Balassiano IT, Yates EA, Domingues RM, et al. Clostridium difficile: a problem of concern in developed countries and still a mystery in Latin America. J Med Microbiol 2012;61(Pt 2):169-79.

2. Guh AY, Mu Y, Baggs J, et al. Trends in incidence of long-term-care facility onset Clostridium difficile infections in 10 US geographic locations during 2011-2015. Am J Infect Control 2018. doi: 10.1016/j.ajic.2017.11.026. [Epub ahead of print 9 Jan 2018].

3. Miller BA, Chen LF, Sexton DJ, et al. Comparison of the burdens of hospital-onset, healthcare facility-associated Clostridium difficile Infection and of healthcare-associated infection due to methicillinresistant Staphylococcus aureus in community hospitals. Infect Control Hosp Epidemiol 2011;32:387-90.

4. Magill SS, Edwards JR, Bamberg W, et al. Multistate pointprevalence survey of health care-associated infections. N Engl $J$ Med 2014;370:1198-208.
5. Dethlefsen L, Huse S, Sogin ML, et al. The pervasive effects of an antibiotic on the human gut microbiota, as revealed by deep $16 \mathrm{~S}$ rRNA sequencing. PLoS Biol 2008;6:e280.

6. Leffler DA, Lamont JT. Clostridium difficile infection. N Engl J Med 2015;372:1539-48.

7. Lessa FC, Mu Y, Bamberg WM, et al. Burden of Clostridium difficile infection in the United States. N Engl J Med 2015;372:825-34.

8. He W, Goodkind D, Kowal P, et al. An aging world: 2015. Washington, DC: U.S. Census Bureau, 2015.

9. Blaser N, Zahnd C, Hermans S, et al. Tuberculosis in Cape Town: an age-structured transmission model. Epidemics 2016;14:54-61.

10. WHO. Global tuberculosis report 2014. Geneva, Switzerland: World Health Organization, 2014

11. UNAIDS. Country factsheets South Africa 2016: UN Joint Programme on HIVIAIDS, 2017.

12. Kwan CK, Ernst JD. HIV and tuberculosis: a deadly human syndemic. Clin Microbiol Rev 2011;24:351-76.

13. South African National AIDS Council. National strategic plan for HIV, TB and STIS (NSP) 2017-2022, 2017.

14. Lehohla PJ. Mortality and causes of death in South Africa, 2015: Findings from death notification. http://www.statssa.gov.za/ publications/P03093/P030932015.pdf

15. Rajabally NM, Pentecost M, Pretorius G, et al. The Clostridium difficile problem: a South African tertiary institution's prospective perspective. S Afr Med J 2013;103:168-72.

16. Rajabally N, Kullin B, Ebrahim K, et al. A comparison of Clostridium difficile diagnostic methods for identification of local strains in a South African centre. J Med Microbiol 2016;65:320-7.

17. Giancola SE, Williams RJ, Gentry CA. Prevalence of the Clostridium difficile BI/NAP1/027 strain across the United States Veterans Health Administration. Clin Microbiol Infect 2017 (published Online First: 2017/11/22).

18. Republic of South Africa. Essential drugs programme. Hospital level (adults) standard treatment guidelines and essential medicines list. 4th ed. Pretoria, South Africa: Republic of South Africa: National Department of Health, 2015.

19. Lee YM, Huh KC, Yoon SM, et al. Incidence and clinical outcomes of clostridium difficile infection after treatment with tuberculosis medication. Gut Liver 2016;10:250-4.

20. Dos Santos-Schaller O, Boisset S, Seigneurin A, et al. Recurrence and death after Clostridium difficile infection: gender-dependant influence of proton pump inhibitor therapy. Springerplus 2016;5:430.

21. Khanafer N, Touré A, Chambrier C, et al. Predictors of Clostridium difficile infection severity in patients hospitalised in medical intensive care. World J Gastroenterol 2013;19:8034-41.

22. World Health Organization. Tuberculosis country profiles [updated 2016]. https://extranet.who.int/sree/Reports?op=Replet\&name=/ WHO_HQ_Reports/G2/PROD/EXT/TBCountryProfile\&ISO2=ZA\& outtype=PDF

23. Baker P, Dworkin SL, Tong S, et al. The men's health gap: men must be included in the global health equity agenda. Bull World Health Organ 2014;92:618-20.

24. Smith AM, Wuerth BA, Wiemken TL, et al. Prevalence of clostridium difficile infection presenting to US EDs. Am J Emerg Med 2015;33:238-43.

25. England PH. Clostridium difficile Ribotyping Network (CDRN) for England and Northern Ireland. 2013 to 2016;2015 https://www. gov.uk/government/uploads/system/uploads/attachment_data/file/ 491253/CDRN_2013-15_Report.pdf.

26. McDonald LC, Gerding DN, Johnson S, et al. Clinical practice guidelines for clostridium difficile infection in adults and children: 2017 update by the Infectious Diseases Society of America (IDSA) and Society for Healthcare Epidemiology of America (SHEA). Clin Infect Dis 2018 (published Online First: 2018/02/15).

27. Stevens VW, Nelson RE, Schwab-Daugherty EM, et al. Comparative effectiveness of vancomycin and metronidazole for the prevention of recurrence and death in patients with clostridium difficile infection. JAMA Intern Med 2017;177:546-53.

28. Nelson RL, Suda KJ, Evans CT. Antibiotic treatment for Clostridium difficile-associated diarrhoea in adults. Cochrane Database Syst Rev 2017;3:Cd004610.

29. Debast SB, Bauer MP, Kuijper EJ, et al. European Society of Clinical Microbiology and Infectious Diseases: update of the treatment guidance document for Clostridium difficile infection. Clin Microbiol Infect 2014;20(Suppl 2):1-26. 